\title{
OPTIMAL EMBEDDINGS OF GENERALIZED INHOMOGENEOUS SOBOLEV SPACES ON $\mathbf{R}^{n}$
}

\author{
IrshaAD AHMED AND GEORGI E. KARADZHOV
}

\begin{abstract}
We prove optimal embeddings in the subcritical case of the inhomogeneous Sobolev spaces built-up over function spaces in $\mathbf{R}^{n}$ with $K$ - monotone and rearrangement invariant norm into another rearrangement invariant function spaces. The investigation is based on pointwise and integral estimates of the rearrangement or the oscillation of the rearrangement of $f$ in terms of the rearrangement of the derivatives of $f$.
\end{abstract}

Mathematics subject classification (2010): 46E35.

Keywords and phrases: Sobolev inhomogeneous spaces, optimal embeddings, rearrangement invariant spaces.

\section{REFERENCES}

[1] C. Bennett, R. Sharpley, Interpolation of operators, Academic Press, New York, 1988.

[2] J. Bastero, M. Milman, F. Ruiz, A note on $L(\infty, q)$ spaces and Sobolev embeddings, Indiana Univ. Math. J. 52 (2003), 1215-1230.

[3] A. Cianchi, Symmetrization and second order Sobolev inequalities, Annali di Matem. 183 (2004), $45-77$.

[4] A. Gogatishvili, V. I. Ovchinnikov, Interpolation orbits and optimal Sobolev's embeddings, J. Funct. Anal. 253 (2007), 1-17.

[5] D. E. Edmunds, W. D. Evans, G. E. Karadzhov, Sharp estimates of the embedding constants for Besov spaces, Rev. Mat. Complut. 19 (2006), 161-182.

[6] D. E. Edmunds, R. Kerman, L. Pick, Optimal Sobolev embeddings involving rearrangement invariant quasinorms, J. Funct. Anal. 170 (2000), 307-355.

[7] D. E. Edmunds, H. TRIEBEL, Sharp Sobolev embeddings and related Hardy inequalities: the critical case, Math. Nachr. 207 (1999), 79-92.

[8] R. Kerman, L. Pick, Optimal Sobolev imbeddings, Forum Math. 18 (2006), 535-579.

[9] V. I. KolyadA, Rearrangements of functions and embedding theorems, Russian Math. Surveys 44 (1989), 73-117.

[10] J. Martín, M. Milman, E. Pustylnik, Sobolev inequalities: Symmetrization and Self improvement via truncation, J. Funct. Anal. 252 (2007), 677-695.

[11] J. Martín, M. Milman, Higher order symmetrization inequalities and applications, J. Math. Anal. and Appl. 330 (2007), 91-113.

[12] M. Milman, E. Pustylnik, On sharp higher order Sobolev embeddings, Comm. Contemp. Math. 6 (2004), 495-511.

[13] J. Vybíral, Optimal Sobolev embeddings on $\mathbf{R}^{n}$, Publ. Mat. 51 (2007), 17-44. 